\title{
Microbial metabolites and graft versus host disease
}

\author{
M. Riwes | P. Reddy
}

Blood and Marrow Transplant Program, Division of Hematology/ Oncology, Department of Internal Medicine, University of Michigan Comprehensive Cancer Center, Ann Arbor, MI, USA

\section{Correspondence}

Pavan Reddy

Email: reddypr@med.umich.edu

Funding information

Foundation for the National Institutes of Health, Grant/Award Number: CA173878, CA203542 and HL128046-02
The health of mammals is a reflection of the diversity and composition of the intestinal microbiota. Alterations in the composition and functions of the intestinal microbiota have been implicated in multiple disease processes. The impact of the microbiota in health and disease is in part a function of the nutrient processing and release of metabolites. Recent studies have uncovered a major role for microbial metabolites in the function of the host immune system by which they influence disease processes such as acute graft versus host disease (GVHD), which is the main complication of allogeneic hematopoietic cell transplantation (allo-HCT). The mechanisms of acute GVHD regulation by the complex microbial community and the metabolites released by them are unclear. In this review we summarize major findings of how microbial metabolites interact with the immune system and discuss how these interactions could impact acute GVHD.

KEYWORDS

basic (laboratory) research/science, bone marrow/hematopoietic stem cell transplantation, graft-versus-host disease (GVHD), intestinal disease: metabolic, translational research/science

\section{1 | INTRODUCTION}

The human body is colonized by communities of microorganisms termed the "microbiota," which include bacteria, fungi, and viruses and the gut is the main site of microbial colonization. ${ }^{1}$ In this review we will focus on the intestinal microbiota. The intestinal microbiota is comprised of trillions of a diverse community of microorganisms largely consisting of nonpathogenic anaerobic commensal bacteria. ${ }^{1}$ In recent years, high throughput sequencing technologies have made it possible to analyze a large number of microorganisms in the gut. Bacteroidetes and Firmicutes are the most prevalent bacterial phyla in the intestine but Actinobacteria, Proteobacteria, and Verrucomicrobia are also present. ${ }^{1}$ The microbiota and the host interact in delicate harmony to create a healthy balanced state and disturbances of this mutualistic symbiotic balance have been linked to a range of diseases including obesity, inflammatory bowel disease, colorectal cancer, and diabetes. ${ }^{1}$
Allogeneic hematopoietic cell transplantation (Allo-HCT) is a potentially curative treatment modality for patients with a variety of malignant and benign hematological diseases. However, the effectiveness of allo-HCT is limited by acute graft versus host disease (GVHD) which is the principal cause of non-relapse mortality. ${ }^{2}$ Conditioning regimens used in allo-HCT cause host tissue injuries which release "danger signals" that activate host or donor antigen presenting cells (APCs) which in turn present allo-antigens via major histocompatibility complex (MHC) class I or class II to donor T cells. ${ }^{3}$ In addition, activated APCs produce T-cell stimulating cytokines which further escalate the inflammatory response. Stimulated donor T cells expand and differentiate into effector $T$ cells which migrate into and damage acute GVHD target organs such as the gastrointestinal (GI) tract. The activation of APCs is amplified by microbial stimulation via pathogenassociated molecular patters (PAMPs) and damage-associated molecular patters (DAMPs).

Abbreviations: AhR, Aryl hydrocarbon receptor; allo-HCT, allogeneic hematopoietic cell transplantation; AMP, antimicrobial peptide; APC, antigen presenting cell; DAMPs, damage-associated molecular patters; DC, dendritic cell; FMT, fecal microbiota tranplant; Foxp3, forkhead box protein; FXR, farnesoid X receptor; GI, gastrointestinal; GPBAR, G protein-coupled bile acid receptor; GPR, G protein-coupled receptor; GVHD, Graft versus host disease; HDAC, histone deacetylase; IEC, intestinal epithelial cell; IEL, intraepithelial lymphocyte; ILC, innate lymphoid cell; ILF, isolated lymphoid follicle; ISC, intestinal stem cells; LPS, lipopolysaccharide; MHC, major histocompatibility complex; MLN, mesenteric lymph node; MYD88, myeloid differentiation primary response protein 88; NF-кB, nuclear factor $\kappa B$; NLR, NOD-like receptor; PAMP, pathogen-associated molecular patter; PP, peyer's patch; PRR, pathogen recognition receptor; ROR $\gamma$ t, retinoic acid receptor-related orphan receptor- $\gamma \mathrm{t}$; SCFA, short chain fatty acid; TGF- $\beta$, transforminggrowth factor-beta; TLR, toll-like receptors; Treg, regulatory T cell. 
The relationship between the intestinal microbiota and acute GVHD has been a topic of interest since the 1970 s following seminal observations by van Bekkum et al in experimental models. ${ }^{4}$ However, scrupulous examination of this relationship has become possible only recently with advances in high throughput sequencing technologies. Emerging data suggest that alterations in the intestinal microbiota and microbial metabolites may play important roles in modulating the severity of acute GVHD. In this review, we summarize these data and recent efforts that investigated the roles of the intestinal microbial metabolites in shaping the host immune system as well as discuss potential new avenues for exploring these roles in intestinal acute GVHD.

\section{2 | INTESTINAL MICROBIOTA}

Herein, we briefly discuss intestinal microbiota and its role in interacting with the immune system and modulating intestinal homeostasis. We would refer readers to recent reviews that have covered this topic in depth. ${ }^{1,5}$ Microbiota-host immune system interactions are important mediators of both tolerance and protection. Maintaining this equilibrium between the host and the microbiome involves the innate and adaptive immune systems as well as other non-immunologic defensive tactics such as the release of antimicrobial peptides (AMPs) and the generation of the mucus barrier. ${ }^{5}$ Many immune cells are involved in this mutualistic interactions, include immune cells isolated lymphoid follicles (ILFs), peyer's patches (PPs), mesenteric lymph nodes (MLNs), and lamina propria immune cells such as macrophages, dendritic cells (DCs), T cells, B cells, and innate lymphoid cells (ILCs). Lamina propria macrophages phagocytose and eliminate bacteria that penetrate the intestinal epithelial cell (IEC) barrier. Lamina propria DCs engulf penetrant bacteria, traffic to secondary lymphoid tissues such as PPs and MLNs, and induce $T$ and $B$ cell responses. In response to commensal bacteria, IECs produce cytokines which promote the development of macrophages and DCs with tolerogenic properties. ILCs are also important for intestinal immune homeostasis and are classified into 3 groups. ${ }^{1,5}$ Retinoic acid receptor-related orphan receptor- $\gamma \mathrm{t}(\mathrm{ROR} \gamma \mathrm{t})^{+}$ group 3 innate lymphoid cells (ILC3s) regulate commensal bacteria by limiting local and systemic inflammation. ROR $\gamma \mathrm{t}^{+}$ILC3s produce the epithelial cell regenerative factor IL-22 in response to IL-23 produced by myeloid cells. Disturbances of immune structures can result in dysbiosis of the intestinal microbiota. For example, the absence of ILFs altered the composition of the intestinal microbiota in mice by over representation of gram negative bacteria. ${ }^{6}$ The intestinal microbiota in turn shapes the innate and adaptive host immune responses. Germ free mice colonized with conventional intestinal microbiota developed innate immune responses and structural changes with increase in the depth of the intestinal epithelial crypts as well as expansion in the lamina propria with an increase in the immune cells within 4 days after exposure to intestinal bacteria. ${ }^{7}$ The intestinal microbiota was also linked to the regulation of adaptive immune cells as intestinal commensal microbes were shown to induce forkhead box protein (Foxp3)- expressing regulatory $T$ cells (Tregs) which produce IL-10, an anti-inflammatory cytokine. ${ }^{8}$ In contrast, stimuli associated with pathogenic bacteria cause inflammatory responses via IL-1 and IL-6 induction which result in Th1 and Th17 activation. ${ }^{1,5}$

Non-immune intestinal cells such as intestinal epithelial cells (IECs), Goblet cells, Paneth cells and intestinal stem cells (ISCs) play important roles in regulating the barrier function of the GI tract and the intestinal microbiota has been shown to promote the functions of these cells. IECs form a continuous physical barrier to separate luminal organisms from intestinal tissues and ISCs play an important role in maintaining the turnover of IECs. ${ }^{1}$ Germ-free mice show slower turnover of IECs compared with conventionally raised mice. ${ }^{7}$ Goblet cells protect the epithelium from luminal bacteria by mucin secretion. ${ }^{1}$ Commensal microbes enhance mucin production by goblet cells while germ-free mice show markedly reduced levels of mucin. Paneth cells secrete AMPs such as $\alpha$-defensins and RegIll $\alpha$ in response to bacteria and their antigens and play a role in shaping the intestinal microbiota.

\section{3 | MICROBIAL METABOLITES}

The intestinal metabolome consists of products from host metabolism, microbial metabolism, and mammalian-microbial co-metabolism. ${ }^{9}$ Microbial metabolites have varying functions in the gut and they include a diverse repertoire of intermediate and end products of both diet-dependent as well as diet-independent intestinal microbiota metabolism. The non-dietary products are generated by metabolism of endogenous compounds produced by microorganisms and the host. Herein we will focus on the diet dependent metabolites which are generated by the breakdown of the host nutrients by the intestinal microbes.

\section{1 | Short chain fatty acids}

Anaerobic commensal bacteria such as Clostridia species produce short chain fatty acids (SCFAs) from fermentation of non-digestible carbohydrates. ${ }^{10}$ SCFAs are the most studied microbial metabolites and include butyrate, acetate, and proprionate. They serve as an energy source for the intestinal microbiota as well as the IECs and are recognized by the host through the $G$ protein-coupled receptors GPR41, GPR43, and GPR109a. Butyrate is an energy source for IECs as colonocytes from germ-free mice were shown to be in an energydeprived state which led to autophagy whereby the cells degraded their own components in order to maintain energy homeostasis and this was reversed by butyrate supplementation. ${ }^{11}$ Besides providing IECs with an energy source, SCFAs are essential for maintaining the integrity of the intestinal mucosal barrier through regulation of functions of goblet cells. ${ }^{12}$ The barrier integrity of IECs was further shown to be maintained by the SCFA acetate as colonization with Bifidobacterium longum protected mice against death induced by a lethal infection by increasing production of acetate and inhibiting translocation of the E. coli O157:H7 Shiga toxin from the gut lumen into the blood. ${ }^{13}$ In addition to their functions in maintaining the health of the barrier surface of the $\mathrm{Gl}$ tract, microbial metabolites play various roles in modulating the innate and adaptive host immune responses 

Non-digestable
carbohydrates<smiles>C=[V]</smiles>

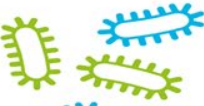
*⿻ers<smiles>CCCC</smiles>
SCFAs

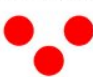

Fatty acids<smiles>C1CC1</smiles>

Dietary polyaimnes, amino acids
Dietary tryptophan<smiles>CCCC</smiles>
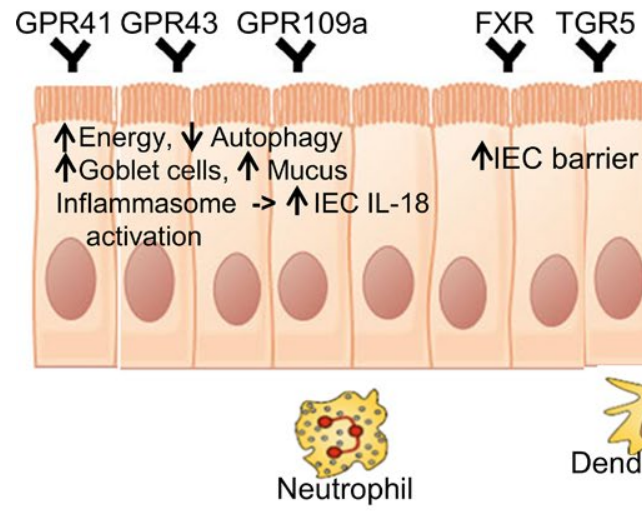

SCFAs

HDACi

$\uparrow$ anti-inflammation

(via NF-kB signaling)

个Treg, 个 IL-10

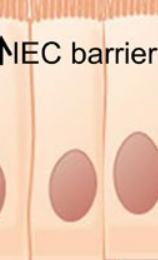

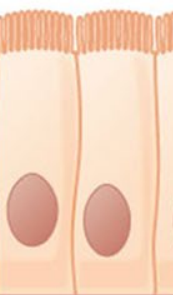

Polya

Microbiota

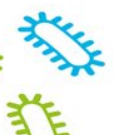

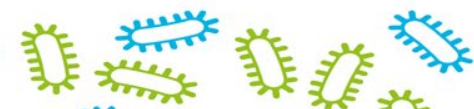
cours<smiles>c1cc2ccc1cc2</smiles>
*⿻一𠃋十<smiles>c1ccc2ccccc2c1</smiles><smiles>C[V]</smiles>

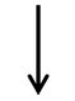

AhR ligands

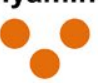

${ }_{0}$

FIGURE 1 Microbial metabolites maintain the health of the intestinal barrier surface and modulate immune responses. Short chain fatty acids (SCFAs) enhance epithelial barrier function and immune tolerance by: serving as an energy source for intestinal epithelial cells (IECs); modulating autophagy; enhancing production of mucus by intestinal Goblet cells; increasing inflammasome activation and subsequent production of IL-18; inhibiting histone deacetylase (HDAC); inhibiting nuclear factor- $\mathrm{KB}(\mathrm{NF}-\mathrm{\kappa B})$; increasing anti-inflammatory effects in macrophages and dendritic cells (DCs); increasing differentiation of regulatory T cells (Tregs) and their production of IL-10. Bile acids enhance epithelial barrier function and inhibit pro-inflammatory cytokines via NF- $\mathrm{kB}$ inhibition. Polyamines augment the barrier integrity of IECs, inhibit pro-inflammatory cytokines and modulate the adaptive immune system by accelerating the maturation of T cell subsets in newborn rats. Aryl hydrocarbon receptor (AhR) ligands enhance the turnover of IECs and expand innate lymphoid cells (ILCs) and their production of the epithelial cell regenerative factor IL-22.

(Figure 1). SCFAs such as butyrate play an integral part in innate immunity as they are histone deacetylase (HDAC) inhibitors that promote an anti-inflammatory cell phenotype. GPCR43 expression was shown to be necessary for the effects of SCFAs on neutrophil chemotaxis. ${ }^{14}$ Other studies showed that SCFAs inhibit HDACs and have anti-inflammatory effects in macrophages and dendritic cells (DCs). ${ }^{15,16}$ In the study by Macia et al, a high fiber diet or treatment with the SCFA acetate in mice increased inflammasome activation and increased levels of IEC protective IL-18 in a GPR43- and GPR109adependent manner which promoted epithelial repair and mitigated the severity of inflammatory colitis. ${ }^{17}$ Besides their involvement in innate immune responses, SCFAs were shown to modulate the adaptive arm of intestinal immunity. Specifically, SCFAs were shown to promote Treg differentiation, promote anti-inflammatory responses and suppress T cell dependent experimental colitis. ${ }^{8}$ Administration of butyrate, acetate, and proprionate to germ-free mice increased the expression of anti-inflammatory IL-10 producing Foxp3-expressing Tregs through HDAC inhibition in a GPR43 dependent manner. ${ }^{5}$ Additionally, butyrate enhanced the expression of IL-18 on IECS and increased IL-10 expression that was critical for protection from colitis in a GPR109a-dependent manner. ${ }^{18}$

\section{2 | Bile acids}

Another group of microbial metabolites that regulate immunity include bile acids. They are synthesized from cholesterol in the liver, conjugated to glycine in humans or taurine in mice, and further metabolized by the intestinal microbiota into secondary bile acids. ${ }^{19}$ Bile acid 
signaling is controlled by the intestinal microbiota through two main receptors: the nuclear farnesoid $X$ receptor $(F X R)$ and the $G$ proteincoupled bile acid receptor 1 (GPBAR1) also known as TGR5. Mice lacking FXR show compromised epithelial barrier function and enhanced bacterial translocation from the intestine. ${ }^{20}$ Bile acids activate TGR5 on macrophages and inhibit production of pro-inflammatory cytokines such as TNF $\alpha$ and II- $1 \beta$ via nuclear factor $\kappa B(N F-\kappa B)$ inhibition. ${ }^{21}$ Furthermore, administration of saturated fatty acids to IL-10 deficient mice altered the composition of the intestinal microbiota and enhanced pro-inflammatory $T_{H^{1}} 1$ response and the severity of colitis. ${ }^{22}$

\section{3 | Polyamines}

Polyamines are polycationic molecules that are found in high concentrations in the Gl tract and are derived from both host and intestinal microbiota metabolism. They have been implicated in a wide range of biological functions. ${ }^{23}$ Importantly, polyamines were shown to augment the barrier integrity of IECs. ${ }^{24}$ They also play a role in innate immunity. The polyamine spermine was shown to inhibit the activation of the pro-inflammatory phenotype of macrophages and suppress the synthesis of pro-inflammatory cytokines without altering the synthesis of anti-inflammatory cytokines transforming growth factor-beta (TGF- $\beta$ ) and IL-10. ${ }^{25}$ Moreover, oral administration of the probiotic bifidobacteria LKM512 in combination with the amino acid arginine, which is metabolized to produce polymaines, increased levels of polyamines, improved longevity, and protected from age-induced memory impairment in mice which correlated with decreased levels of pro-inflammatory cytokines. ${ }^{26}$ Polyamines also modulate adaptive immunity as the oral supplementation of the polyamines spermine or spermidine to newborn rats accelerated the maturation of intraepithelial $\mathrm{CD}^{+} \mathrm{T}$ cells, increased the percentage of lamina propria $\mathrm{CD} 4^{+} \mathrm{T}$ cells, and enhanced B cells in the spleen. ${ }^{27}$

\section{4 | Aryl hydrocarbon receptor ligands}

Aryl hydrocarbon receptor (AhR) is a ligand-activated transcription factor and a modulator of mucosal immune responses. AhR ligands are derived from intestinal microbiota metabolism. The commensal Lactobacilli utilizes amino acid tryptophan to produce AhR ligands such as indole-3-aldehyde. ${ }^{28}$ AhR ligands are critical for the maintenance of the intestinal epithelial barrier. Their deficiency in mice results in alterations of the intestinal microbiota composition with bacterial overgrowth, decrease in AMPs and intestinal epithelial lymphocytes (IELs), and reduced turnover of IECs. ${ }^{29}$ AhR directly regulates innate immunity. It is necessary for expansion of IL-22 producing ROR $y \mathrm{t}^{+}$ILC3s and the formation of ILFs, which were needed for the clearance of Citrobacter rodentium infection. ${ }^{30}$ AhR effect on ROR $\gamma \mathrm{t}^{+}$ILC3s can be induced by dietary ligands such as those contained in vegetables of the family Brassicaceae. ${ }^{30}$ They were also shown to be important in adaptive immunity. A reduction in AhR ligands in response to Cyp1a1 enzyme activity in the IECs decreased Th17 cells and impaired II-22 production in response to enteric infections. ${ }^{31}$

\section{INTESTINAL MICROBIOTA AND ACUTE GVHD}

A healthy intestinal microbiota is diverse and dominated by obligate anaerobic bacteria. ${ }^{1}$ During the process of allo-HCT patients lose this diversity and experience alterations in community composition which are influenced by antibiotic treatment and the development of acute GVHD. ${ }^{32-34}$ Holler et al showed a shift towards Enterococci after allo-HCT with a decrease in the obligate anaerobic bacteria from the phylum Frimicutes that was pronounced in those that were treated with antibiotics. ${ }^{32}$ Thus the process of allo-HCT has been shown to alter the intestinal microbiota composition and affect the intestinal homeostasis.

Reciprocally, the intestinal microbiota has been shown to modulate post allo-HCT outcomes including acute GVHD. ${ }^{35-38}$ Studies from the 1970s investigated the role of the intestinal mcirobiota in allo-HCT and found less acute GVHD in mice transplanted in germfree conditions or receiving gut decontamination antibiotics. ${ }^{4}$ Some follow-up clinical studies showed similar beneficial effects of bacterial decontamination in allo-HCT patients while a few others did not. ${ }^{39-}$

42 The reasons for these disparate results remain unclear but could be due to variation in success of decontamination techniques or the specifics of the alteration in the microbiota composition rather than just a global loss of diversity. Recent studies show that changes in microbial composition post allo-HCT were more pronounced in those that developed acute GVHD including greater loss of diversity, expansion of Enterobacteriales, Lactobacillales, Proteobacteria, and Akkermansia. ${ }^{43,44}$ This was accompanied by the loss of obligate anaerobic bacteria from the phylum Firmicutes (including Clostridia and Blautia). High intestinal abundance of Blautia correlated with lower rates of acute GVHD and improved survival. ${ }^{36}$ It was recently shown that administration of a cocktail of 17 species of Clostridia reduced experimental acute GVHD and improved survival. ${ }^{45}$ These data suggest that changing the microbial architecture in the right manner may be a potentially novel approach for ameliorating acute GVHD. In line with this notion, treating acute GVHD in allo-HCT patients with fecal microbiota transplant (FMT) is currently being investigated in clinical trials (www.clinicaltrials.gov: NCT02269150, NCT02733744). Of interest, recent very small studies suggested that FMT after allo-HCT is safe and may have clinical impact in patients with advanced acute GI GVHD. ${ }^{46-48}$

Most of the aforementioned studies of the effect of the microbiota on acute GVHD are based on associations but the causations and potential mechanisms of how the microbiota modulates acute GVHD severity remain unknown. Host microbial sensing by pathogen recognition receptors (PRRs) has been suggested to play a role in modulating experimental acute GVHD. ${ }^{3}$ For example donor cell responses to PAMPs, such as lipopolysaccharides (LPS), amplifies experimental acute GVHD. ${ }^{3}$ In humans, TLR4 polymorphisms were associated with lower risk of acute GVHD. ${ }^{43}$ TLR9 was shown to play a role in an experimental model of acute GVHD. Similarly, NOD2 was reported to be associated with acute experimental and clinical acute GVHD. ${ }^{3}$ The role of PRRs in acute GVHD has been covered in other recent reviews. ${ }^{3}$ 
Nonetheless the PRRs do not completely allow for understanding the changes in microbial communities and their causal role, if any, in acute GVHD severity.

\section{5 | MICROBIAL METABOLITES AND ACUTE GVHD}

The changes in microbiome structure cause a change in intestinal metabolites. A recent study explored the role of microbial metabolites in acute GVHD severity. ${ }^{45}$ The study reported data primarily on SCFAs and long chain fatty acids (LCFAs) from skin, intestine and liver post experimental allo-HCT. The study demonstrated that amongst all the SCFAs and LCFAs from various tissues and stool, only the SCFA butyrate was significantly decreased only in the IECs. The impact on non fatty acid metabolites was not reported. The reduction in the IEC butyrate was associated with reduction in the microbial diversity and in the family of bacteria that are known to produce butyrate from resistant starch. An interesting aspect of the study was that the diet was kept constant. It remains unknown if changing the diet to resistant starch, the fodder for bacterial species that break it down to butyrate, might alter the butyrate. However, the study reported that supplementing the microbiome with 17 strains of clostridial species that are known to be butyrate producers restored butyrate levels in both antibiotic treated or untreated recipients. Furthermore, the restoration of butyrate levels with exogenous administration of butyrate without changing the microbiome promoted histone acetylation in IECs and caused enhanced expression of anti-apoptotic proteins involved in barrier integrity such as JAM and occludin. Importantly, butyrate administration increased the expression of its surface receptor, GPR43 and transporter protein, SLC5A8. Most relevantly, restoring butyrate levels, either by direct administration of butyrate or by changing the microbiome with increasing butyrogenic bacteria such as with the 17 strains of Clostridia, mitigated intestinal barrier damage, reduced acute GVHD specific damage, and improved survival after allo-HCT. Mechanistically, butyrate increased histone acetylation and was also rapidly metabolized by the IECs after allo-HCT. Ex vivo studies showed that the IECs, upon treatment with SCFA butyrate, showed a reduction with apoptosis when cultured with alloreactive T cells. Intriguingly, the reduction in acute GVHD was observed even when the donor Tregs were depleted from the donor cells. While these data suggest protective effects on acute GVHD could occur independent of donor Tregs, the study did not explore whether host Tregs played a role in acute GVHD protection. However, the report suggests that direct effect of butyrate on IECs might play a role in reducing acute GVHD severity. This concept is now being studied in two clinical trials (www.clinicaltrials.gov: NCT02763033, NCT02805075). Of interest, one small study in 20 healthy young adults showed that potato-based starches induce production of butyrate from the microbiota. ${ }^{49}$ A clinical trial of this prebiotic aimed at increasing butyrate levels and prevention of acute GVHD is underway (www.clinicaltrials.gov: NCT02763033).
TABLE 1 Microbial metabolites involved in maintaining the health of the intestinal barrier surface and modulating immune responses and their known roles in acute graft versus host disease (GVHD)

\begin{tabular}{|c|c|}
\hline Microbial metabolites & Roles explored in acute GVHD \\
\hline Short chain fatty acids (SCFA) & $\begin{array}{l}\text { Direct effect of the SCFA butyrate } \\
\text { on intestinal epithelial cells (IECS): } \\
\text { reduced severity of experimental } \\
\text { intestinal acute GVHD }\end{array}$ \\
\hline Bile acids & To be determined \\
\hline Polyamines & To be determined \\
\hline $\begin{array}{l}\text { Aryl hydrocarbon receptor } \\
\text { (AhR) ligands }\end{array}$ & $\begin{array}{l}\text { Low levels of urine } 3 \text {-indoxyl } \\
\text { sulfate correlated with increased } \\
\text { clinical intestinal acute GVHD }\end{array}$ \\
\hline
\end{tabular}

Currently there is paucity of data on other intestinal microbial metabolites and their impact in acute GVHD (Table 1). However, one clinical study in allo-HCT patients showed that lower levels of urine 3-indoxyl sulfate, a metabolite derived from indole produced by commensal bacteria and an AhR ligand, correlated with higher treatment related mortality and lower overall survival. ${ }^{50}$

\section{6 | FUTURE PERSPECTIVES}

The aforementioned recent study which explored the role of microbial metabolites in acute GVHD severity ${ }^{45}$ raises several interesting questions including, but not limited to, whether it is possible to mitigate GI damage by directly affecting target cells without substantially affecting the immune system. ${ }^{51}$ Do other SCFAs a role or is this unique to butyrate? If so, then is butyrate protective primarily by inhibiting HDACs and/or by providing energy to the IECs under duress from inflammation? This study demonstrated that microbial metabolites are critical and suggest these are relevant causal mediators of the microbial changes associated with acute GVHD severity. ${ }^{45}$ It opens up questions for the putative roles of other metabolites, their correlation and relationship to the dynamics of microbiome alterations, the role of sensors of these metabolites, the impact of these on immune system, on IECs, ISCs, Paneth cells, and other tissues in acute GVHD. Of particular interest, AhR was necessary for expansion of IL-22 producing ILCs. ${ }^{30}$ IL-22 is an epithelial cell regenerative factor and IL-22 producing ILCs were found to be depleted in the intestines of mice with acute GVHD and treatment with IL-22 enhanced the recovery of ISCs, increased epithelial regeneration, and reduced acute GVHD. ${ }^{52}$ Administration of IL-22 along with systemic corticosteroids is now being investigated in a clinical trial for initial treatment of grade II-IV lower GI acute GVHD (www.clinicaltrials.gov: NCT02406651), but the role that AhR plays in modulating IL-22 producing ILCs in acute GVHD and whether dietary ligands can modulate that role are yet to be investigated. Additionally, several microbial metabolites can impact adaptive immune responses and promote Treg differentiation ${ }^{5,8,18}$, which is of particular interest given that Tregs suppress acute GVHD ${ }^{2}$ and warrants further investigation. In summary, more investigations of the role of microbial metabolites in GVHD are needed. 


\section{DISCLOSURE}

The authors of this manuscript have no conflicts of interest to disclose as described by the American Journal of Transplantation.

\section{REFERENCES}

1. Hooper LV, Littman DR, Macpherson AJ. Interactions between the microbiota and the immune system. Science. 2012;336:1268-1273.

2. Choi SW, Reddy P. Current and emerging strategies for the prevention of graft-versus-host disease. Nat Rev Clin Oncol. 2014;11:536-547.

3. Toubai T, Mathewson ND, Magenau J, Reddy P. Danger signals and graft-versus-host disease: current understanding and future perspectives. Front Immunol. 2016;7:539.

4. van Bekkum DW, Roodenburg J, Heidt PJ, van der Waaij D. Mitigation of secondary disease of allogeneic mouse radiation chimeras by modification of the intestinal microflora. J Natl Cancer Inst. 1974;52:401-404.

5. Smith PM, Howitt MR, Panikov N, et al. The microbial metabolites, short-chain fatty acids, regulate colonic Treg cell homeostasis. Science. 2013;341:569-573.

6. Bouskra D, Brezillon C, Berard M, et al. Lymphoid tissue genesis induced by commensals through NOD1 regulates intestinal homeostasis. Nature. 2008;456:507-510.

7. El Aidy S, van Baarlen P, Derrien M, et al. Temporal and spatial interplay of microbiota and intestinal mucosa drive establishment of immune homeostasis in conventionalized mice. Mucosal Immunol. 2012;5:567-579.

8. Atarashi $\mathrm{K}$, Tanoue $\mathrm{T}$, Oshima $\mathrm{K}$, et al. Treg induction by a rationally selected mixture of Clostridia strains from the human microbiota. Nature. 2013;500:232-236.

9. Backhed F, Ley RE, Sonnenburg JL, Peterson DA, Gordon JI. Host-bacterial mutualism in the human intestine. Science. 2005;307:1915-1920.

10. Koh A, De Vadder F, Kovatcheva-Datchary P, Backhed F. From dietary fiber to host physiology: short-chain fatty acids as key bacterial metabolites. Cell. 2016;165:1332-1345.

11. Donohoe DR, Garge N, Zhang X, et al. The microbiome and butyrate regulate energy metabolism and autophagy in the mammalian colon. Cell Metab. 2011;13:517-526.

12. Willemsen LE, Koetsier MA, van Deventer SJ, van Tol EA. Short chain fatty acids stimulate epithelial mucin 2 expression through differential effects on prostaglandin $\mathrm{E}(1)$ and $\mathrm{E}(2)$ production by intestinal myofibroblasts. Gut. 2003;52:1442-1447.

13. Fukuda $\mathrm{S}$, Toh $\mathrm{H}$, Hase $\mathrm{K}$, et al. Bifidobacteria can protect from enteropathogenic infection through production of acetate. Nature. 2011;469:543-547.

14. Maslowski KM, Vieira AT, $\mathrm{Ng} \mathrm{A}$, et al. Regulation of inflammatory responses by gut microbiota and chemoattractant receptor GPR43. Nature. 2009:461:1282-1286.

15. Chang PV, Hao L, Offermanns S, Medzhitov R. The microbial metabolite butyrate regulates intestinal macrophage function via histone deacetylase inhibition. Proc Natl Acad Sci USA. 2014;111:2247-2252.

16. Singh N, Thangaraju M, Prasad PD, et al. Blockade of dendritic cell development by bacterial fermentation products butyrate and propionate through a transporter (SIc5a8)-dependent inhibition of histone deacetylases. J Biol Chem. 2010;285:27601-27608.

17. Macia L, Tan J, Vieira AT, et al. Metabolite-sensing receptors GPR43 and GPR109A facilitate dietary fibre-induced gut homeostasis through regulation of the inflammasome. Nat Commun. 2015;6:6734.

18. Singh N, Gurav A, Sivaprakasam S, et al. Activation of Gpr109a, receptor for niacin and the commensal metabolite butyrate, suppresses colonic inflammation and carcinogenesis. Immunity. 2014;40: 128-139.
19. Ridlon JM, Kang DJ, Hylemon PB. Bile salt biotransformations by human intestinal bacteria. J Lipid Res. 2006;47:241-259.

20. Inagaki T, Moschetta A, Lee YK, et al. Regulation of antibacterial defense in the small intestine by the nuclear bile acid receptor. Proc Nat Acad Sci USA. 2006;103:3920-3925.

21. Pols TW, Nomura M, Harach T, et al. TGR5 activation inhibits atherosclerosis by reducing macrophage inflammation and lipid loading. Cell Metab. 2011;14:747-757.

22. Devkota S, Wang Y, Musch MW, et al. Dietary-fat-induced taurocholic acid promotes pathobiont expansion and colitis in II10-/- mice. Nature. 2012;487:104-108.

23. Di Martino ML, Campilongo R, Casalino M, Micheli G, Colonna B, Prosseda G. Polyamines: emerging players in bacteria-host interactions. Int J Med Microbiol. 2013;303:484-491.

24. Liu L, Guo X, Rao JN, et al. Polyamines regulate E-cadherin transcription through c-Myc modulating intestinal epithelial barrier function. Am J Physiol Cell Physiol. 2009;296:C801-C810.

25. Zhang M, Wang H, Tracey KJ. Regulation of macrophage activation and inflammation by spermine: a new chapter in an old story. Crit Care Med. 2000;28(4 Suppl):N60-N66.

26. Kibe R, Kurihara S, Sakai Y, et al. Upregulation of colonic luminal polyamines produced by intestinal microbiota delays senescence in mice. Sci Rep. 2014:4:4548.

27. Perez-Cano FJ, Gonzalez-Castro A, Castellote C, Franch A, Castell M. Influence of breast milk polyamines on suckling rat immune system maturation. Dev Comp Immunol. 2010;34:210-218.

28. Zelante T, lannitti RG, Cunha C, et al. Tryptophan catabolites from microbiota engage aryl hydrocarbon receptor and balance mucosal reactivity via interleukin-22. Immunity. 2013;39:372-385.

29. Li Y, Innocentin S, Withers DR, et al. Exogenous stimuli maintain intraepithelial lymphocytes via aryl hydrocarbon receptor activation. Cell. 2011;147:629-640.

30. Kiss EA, Vonarbourg C, Kopfmann S, et al. Natural aryl hydrocarbon receptor ligands control organogenesis of intestinal lymphoid follicles. Science. 2011;334:1561-1565.

31. Schiering C, Wincent E, Metidji A, et al. Feedback control of AHR signalling regulates intestinal immunity. Nature. 2017;542: 242-245.

32. Holler E, Butzhammer P, Schmid K, et al. Metagenomic analysis of the stool microbiome in patients receiving allogeneic stem cell transplantation: loss of diversity is associated with use of systemic antibiotics and more pronounced in gastrointestinal graft-versus-host disease. Biol Blood Marrow Transplant. 2014;20:640-645.

33. Shono Y, Docampo MD, Peled JU, et al. Increased GVHD-related mortality with broad-spectrum antibiotic use after allogeneic hematopoietic stem cell transplantation in human patients and mice. Sci Transl Med. 2016;8:339ra371.

34. Weber D, Oefner PJ, Dettmer K, et al. Rifaximin preserves intestinal microbiota balance in patients undergoing allogeneic stem cell transplantation. Bone Marrow Transplant. 2016;51:1087-1092.

35. Taur Y, Jenq RR, Perales MA, et al. The effects of intestinal tract bacterial diversity on mortality following allogeneic hematopoietic stem cell transplantation. Blood. 2014;124:1174-1182.

36. Jenq RR, Taur Y, Devlin SM, et al. Intestinal blautia is associated with reduced death from graft-versus-host disease. Biol Blood Marrow Transplant. 2015;21:1373-1383.

37. Peled JU, Devlin SM, Staffas A, et al. Intestinal microbiota and relapse after hematopoietic-cell transplantation. J Clin Oncol. 2017;35:1650-1659.

38. Bilinski J, Robak K, Peric Z, et al. Impact of gut colonization by antibiotic-resistant bacteria on the outcomes of allogeneic hematopoietic stem cell transplantation: a retrospective single-center study. Biol Blood Marrow Transplant. 2016;22:1087-1093.

39. Storb R, Prentice RL, Buckner CD, et al. Graft-versus-host disease and survival in patients with aplastic anemia treated by marrow grafts 
from HLA-identical siblings. Beneficial effect of a protective environment. N Engl J Med. 1983;308:302-307.

40. Russell JA, Chaudhry A, Booth K, et al. Early outcomes after allogeneic stem cell transplantation for leukemia and myelodysplasia without protective isolation: a 10-year experience. Biol Blood Marrow Transplant. 2000;6:109-114.

41. Vossen JM, Guiot HF, Lankester AC, et al. Complete suppression of the gut microbiome prevents acute graft-versus-host disease following allogeneic bone marrow transplantation. PLOS ONE. 2014;9: e105706.

42. Routy B, Letendre C, Enot D, et al. The influence of gutdecontamination prophylactic antibiotics on acute graft-versus-host disease and survival following allogeneic hematopoietic stem cell transplantation. Oncoimmunology. 2017;6:e1258506.

43. Staffas A, Burgos da Silva M, Van den Brink MR. Brink MR. The intestinal microbiota in allogeneic hematopoietic cell transplant and graftversus-host disease. Blood. 2017;129:927-933.

44. Jenq RR, Ubeda C, Taur Y, et al. Regulation of intestinal inflammation by microbiota following allogeneic bone marrow transplantation. J Exp Med. 2012;209:903-911.

45. Mathewson ND, Jenq R, Mathew AV, et al. Gut microbiome-derived metabolites modulate intestinal epithelial cell damage and mitigate graft-versus-host disease. Nat Immunol. 2016;17:505-513.

46. Kakihana K, Fujioka Y, Suda W, et al. Fecal microbiota transplantation for patients with steroid-resistant acute graft-versus-host disease of the gut. Blood. 2016;128:2083-2088.
47. Spindelboeck W, Schulz E, Uhl B, et al. Repeated fecal microbiota transplantations attenuate diarrhea and lead to sustained changes in the fecal microbiota in acute, refractory gastrointestinal graft-versushost-disease. Haematologica. 2017;102:e210-e213.

48. Webb BJ, Brunner A, Ford CD, Gazdik MA, Petersen FB, Hoda D. Fecal microbiota transplantation for recurrent Clostridium difficile infection in hematopoietic stem cell transplant recipients. Transpl Infect Dis. 2016;18:628-633.

49. Venkataraman A, Sieber JR, Schmidt AW, Waldron C, Theis KR, Schmidt TM. Variable responses of human microbiomes to dietary supplementation with resistant starch. Microbiome. 2016;4:33.

50. Weber D, Oefner PJ, Hiergeist A, et al. Low urinary indoxyl sulfate levels early after transplantation reflect a disrupted microbiome and are associated with poor outcome. Blood. 2015;126:1723-1728.

51. Wu SR, Reddy P. Tissue tolerance: a distinct concept to control acute GVHD severity. Blood. 2017;129:1747-1752.

52. Lindemans CA, Calafiore M, Mertelsmann AM, et al. Interleukin-22 promotes intestinal-stem-cell-mediated epithelial regeneration. Nature. 2015;528:560-564.

How to cite this article: Riwes M, Reddy P. Microbial metabolites and graft versus host disease. Am J Transplant. 2018;18:23-29. https://doi.org/10.1111/ajt.14443 